\title{
Patients, doctors and experimentation: doubts about the Declaration of Helsinki
}

\author{
Andrew Belsey Department of Philosophy, University College, Cardiff
}

\begin{abstract}
The World Medical Association's Declaration of

Helsinki offers guidelines to doctors engaged in biomedical research with human subjects. The fundamental distinction of the Declaration is between clinical research combined with professional care and non-clinical scientific research. If hospital patients are the experimental subjects, then the former research must be carried out by the patient's own doctor, whereas the latter research must not be; it must be carried out by other doctors. The relevance of the distinction between the patient's own doctor and other doctors is challenged and an alternative conception of the patient-doctor relationship is drawn, together with an alternative justification, based on the work of Hans fonas, of the use of patients in research. Finally, the political nature of the patient-doctor relationship is mentioned, and it is claimed that the alternative conception of this relationship developed in the paper is more in line with the ethical principles of the Hippocratic tradition.
\end{abstract}

Life is short, science is long; opportunity is elusive, experiment is dangerous, judgement is difficult. It is not enough for the physician to do what is necessary, but the patient and the attendants must do their part as well, and circumstances must be favourable.

Hippocrates $^{1}$

\section{Introduction}

The Declaration of Helsinki is in general a useful and valuable document, setting the high ethical standards necessary to prevent the degeneration of biomedical research from its humanitarian purpose. ${ }^{2}$ There is a point or two which the critic, if wishing to be severe, could call an inconsistency, or if kinder, merely a minor drafting difficulty - for example, paragraph II 5 recognises that there may be cases in clinical research in which informed consent should not be obtained, whereas paragraph I 9 (of 'Basic Principles') apparently does not envisage the possibility of any such cases, since it says that informed consent should be obtained in any research on human beings.

But in only one area is there, I suggest, the necessity for serious rethinking. This concerns the use of patients in research. The Declaration, I believe, is based on an inadequate conception of the patient-doctor relationship, especially as far as the hospital in-patient is concerned, and further, it does not notice the political, and hence contested, nature of this relationship. These points I shall attempt argue for in the rest of this paper.

\section{Two types of research}

It is central to the Declaration that there is distinction between clinical research and now clinical research:

In the field of biomedical research a fundament distinction must be recognised between medic research in which the aim is essentially diagnostic of therapeutic for a patient, and medical research, th essential object of which is purely scientific and without direct diagnostic or therapeutic value to the person subjected to the research (Introduction, paragraph 6).

Clinical research is therapeutic research, researci combined with professional care, and hence carrieg out by the doctor treating the patient. It must related to the patient's illness and be likely benefit that patient; it must not be used just for the acquisition of useful knowledge. Non-clinicad research is non-therapeutic, not combined wit professional care, and done for the scientific purpose of advancing knowledge in medicine or biology.

Clearly, the former research must be on patientis and therefore it must also be, as the Declaration puts it, medical research combined with professiona care (section II, title). Now the Declaration accep that a doctor must be free to use new, and therefo experimental, measures if they are likely to benefit the patient without undue risk (paragraphs II 1,29 Such research may well of course, provide knowledge that could be applied for the general good, but the Declaration is quite clear that a doctor must not experiment on a patient solely to acquire knowledgev

The doctor can combine medical research with professional care, the objective being the acquisition of new knowledge, only to the extent that medic research is justified by its potential diagnostic or therapeutic value for the patient (paragraph II 6).

So far, then, the Declaration seems quite clear. I line with the traditional patient-doctor relationshi帒 of medical ethics, a doctor must not use a patient for research that is not likely to benefit the patient.

However, when the Declaration turns to non? clinical biomedical research, or what it calls 'the 
purely scientific application of medical research carried out on a human being' (paragraph III I), it says the following:

The subjects should be volunteers - either healthy persons or patients for whom the experimental design is not related to the patient's illness (paragraph III 2).

So a doctor may experiment on a patient, provided that the research is not related to the patient's illness and could not therefore, benefit the patient!

\section{Is this inconsistent?}

Is there an inconsistency here, or can it be resolved ? Yes, it can be resolved, but only by drawing a distinction between the patient's own doctor and other doctors. If medical research is combined with professional care, then this research would have to be carried out by the doctor who is caring for the patient, the patient's own doctor. So when the research was purely scientific, it would have to be carried out by a doctor who was not involved in professional care, who was not the patient's own doctor. Therefore, what is implied in the Declaration is that the patient's own doctor may not experiment on him or her for non-clinical reasons, but other doctors may. So there need be no inconsistency. Nevertheless, is what is allowed by the Declaration in fact ethically acceptable?

\section{Jonas's answer}

Its acceptability would be in doubt in a system of ethical principles that drew a tight boundary around the allowable area of experimentation with human beings. For example, Jonas has suggested that the 'spontaneous answer' to the question of who should make themselves available for nonclinical experimentation is

Least and last of all the sick. ... That the afflicted should not be called upon to bear additional burden and risk, that they are society's special trust and the physician's trust in particular - these are elementary responses of our moral sense. ${ }^{3}$

That such responses would severely limit experimentation on patients is apparent (though as I shall mention towards the end of my discussion, Jonas is in one important way more liberal than the Declaration). The basic argument is well-known: those who are ill are less able to give informed consent to experimentation upon themselves because of their disabilities and their relationship of dependence on the doctor.

\section{Patients and doctors}

However, there is another argument that I shall offer, which depends on challenging the relevance of the distinction implicit in the Declaration between $\underset{F}{\stackrel{T}{F}}$ the patient's own doctor and other doctors. I shall $\bar{\delta}$. discuss this with reference to what are the most obvious cases, namely, hospital in-patients, though $\stackrel{9}{\rightarrow}$ no doubt much of what I say will apply in part also $\bar{c}$ to out-patients and patients of general practitioners. 듬

Consider a patient occupying a hospital bed. The $\frac{\bar{p}}{\bar{D}}$ Declaration allows clinical experimentation by the $\mathbb{\otimes}$ patient's own doctor only if combined with pro- on fessional care, and only to the extent that the experimentation is likely to benefit the patient. On the other hand the Declaration allows non-clinical biomedical research on patients, provided it is not carried out by the patient's own doctor.

But for the hospital patient this distinction ought to be challenged. There is first of all the problem of informed consent. The Declaration recognises the general problem when it says:

When obtaining informed consent for the research project the doctor should be particularly cautious if the subject is in a dependent relationship to him or her or may consent under duress. In that case the informed consent should be obtained by a doctor who is not engaged in the investigation and who is completely independent of this official relationship (paragraph I 10).

But the hospital patient is likely to feel under an obligation to anyone in a long white coat who comes along and asks for participation in an experiment; indeed, may well feel less able to resist a request from a strange doctor than one from the doctor who is familiar to the patient. So it is not at all clear that informed consent can be obtained by a doctor who has not been treating the patient and who wishes to use the patient in research unconnected with the patient's illness.

The patient is in fact dependent on a large team: administrators, medical personnel, nursing staff, ancillary workers, and so it is not surprising if he or she feels dependent on the hospital staff as a whole. Further to this, the entire staff has a responsibility to each patient and not just to particular $\frac{\text { 의 }}{7}$ patients. This surely applies strongly to the medical $\frac{D}{O}$ personnel. Any doctor might be called upon if complications arise or in an emergency to render $N$ assistance to a patient. It is true that there are some unlikely combinations: a gynaecologist and a 70-year-old man, for example. But to such a $\omega$ counter-example there are two replies. First, doctors are not just specialists, and it is possible to think of emergency situations in which even a $\Phi$ gynaecologist would be called on to give general $\stackrel{+}{+}$ assistance. But second, just as it is unlikely that a $70-\frac{7}{0}$ year-old man would be a patient of a gynaecologist, $\frac{O}{\mathbb{D}}$ so it is unlikely that he would be an experimental $\frac{\rho}{1}$ subject of the gynaecologist. But anyone who is a $\stackrel{\mathbb{Q}}{\varrho}$ potential experimental subject of a doctor is a potential patient of that doctor, and this is enough to 8 challenge the distinction between the patient's own 
doctor and other doctors. The patient is in the care of, and is the responsibility of, many doctors, who have to work together and offer both their specialised and their general skills when called upon. Therefore the idea that the hospital patient must not be experimented on for non-clinical reasons by his or her own doctor, but is available to other doctors, is doubtful.

\section{Updating Hippocrates}

Challenging the distinction between the patient's own doctor and other doctors has considerable consequences, for it challenges the Hippocratic tradition of a one-to-one relationship between doctor and patient. But I suggest that this ought to be challenged, especially as far as the hospital inpatient is concerned, because it is unrealistic. If it is unrealistic, it would be foolish to base a code of ethics upon it. Of course, the ethics themselves are not challenged. The need for high ethical standards remains, independently of how the patient-doctor relationship is understood.

\section{Rethinking the relationship}

But would not what I have said so far have dire consequences for medical research and therefore for treatment, by making much or all research on hospital patients unethical? Indeed it would, but such an unwanted consequence can be avoided by rethinking the patient-doctor relationship and those parts of the Declaration which depend on the distinction between the patient's own doctor and other doctors.

The fundamental distinction of the Declaration is a two-fold distinction between:

I) Clinical research combined with professional care, 2) Non-clinical biomedical research.

In place of this I shall mention the three-fold distinction put forward by Jonas between:

I) Research related to the patient's illness which is likely to benefit the patient,

2) Research related to the patient's illness which is unlikely to benefit the patient but which is likely to benefit other patients suffering from the same illness,

3) Research unrelated to the patient's illness. ${ }^{4}$

Jonas sees the first category as unproblematic, would rule out the third completely, but is prepared to allow the permissibility of the second in some cases. This is because:

the only excuse for infracting the special exemption of the sick at all - namely, that the scientific war on disease cannot accomplish its goal without drawing the sufferers from disease into the investigative process. $^{5}$
It follows that implicit in Jonas's approach is rejection of the idea that clinical research must be combined with professional care, that is, with. measures likely to benefit the patient; Jonas clearly rejects this in cases in which such measures have been exhausted. ${ }^{6}$

But the rejection of this idea also follows fronts what I have said, because I have suggested that eac

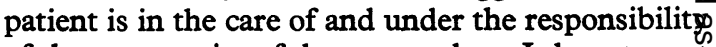
of the community of doctors, and yet I do not want to say that no research by doctors involving patients is permissible. But rethinking the limits of per missibility goes together with rethinking the patiente doctor relationship, and I have suggested, wit considerable help from Jonas, one possible way of doing this. It would probably make any difficulties about informed consent even more intractable than before, but then this is a practical problem that an code of medical ethics must learn to live with. Apare from this, the ethical appraisal of research involving patients is, I conclude, a more subtle task that is recognised by the Declaration of Helsinki. It unable to recognise this, basically because it is base $\overline{8}$ on an unrealistic account of the patient-docto甲 relationship in a modern hospital.

\section{Political implieations}

There is one important consequence of what have said which should be mentioned. If the nature of the patient-doctor relationship is relevant to setting the limits of permissible experimentation on patients, then it should be realised that th $\overrightarrow{\hat{\theta}}$ nature of this relationship is a political issue, an $\bar{\Phi}$ hence contested. I have been writing from the poin of view of what some people insist on calling. 'socialised medicine' - the view which underlies the foundation of the British National Health Service and which is based on recognising the right of the best health care for all, independent of means and influence. Such a recognition makes possible patient-doctor relationships based on ethical principles: trust, care, dedication, humanity - though $\$$ am far from suggesting that such relationships have yet been fully realised. But this is quite differene from the point of view of private medicine, which sees health care as a commodity, and hences must regard the patient-doctor relationship ap fundamentally a financial and not an ethical one. $\omega$

The advocates of private medicine have confuseck the indeed desirable Hippocratic principle of personal medical relationship between patient ans doctor with a personal cash relationship. But I have suggested going beyond challenging this suppose ${ }^{\circ}$ connection between medicine and money and rethinking the patient-doctor relationship so thaf each patient is in the care of the community of doctors. But this is quite compatible with persona? patient-doctor relationships, and in fact most of the time and in normal circumstances a patient would 
be treated by a particular doctor. But the conception of the patient-doctor relationship I have put forward is, I suggest, not only more realistic but involves the best chance of comprehensive medical care being available within the devoted humanitarian principles of the Hippocratic tradition.

\section{Acknowledgements}

The author gratefully acknowledges the assistance of the British Medical Association who kindly supplied a copy of the revised Declaration.

\section{References}

${ }^{1}$ Lloyd, G E R (ed) (1978). Aphorisms, I, I, in Hippocratic Writings, p. 206. Harmondsworth, Penguin.
World Medical Association, Declaration of Helsinki: Recommendations guiding Medical Doctors in Biomedical Research involving Human Subjects (Adopted by the I8th World Medical Assembly, Helsinki, Finland, 1964, and as revised by the 29th World Medical Assembly, Tokyo. Japan, 1975). The Declaration is in four parts: Introduction: I Basic Principles; II Medical Research combined with Professional Care (Clinical Research); III Non-Therapeutic Biomedical Research involving Human Subjects (Non-Clinical Biomedical Research).

JJonas, Hans (1972), Philosophical Reflections on Experimenting with Human Subjects, in Experimentation with Human Subjects, Freund, Paul A. (ed) pp. I-3I at 2I. London. Allen and Unwin.

Ibid, pp. 24-26.

5 Ibid, p. 24.

Ibid, p. 25. 